\title{
Survey of UK computerised special needs registers
}

\author{
Tom Hutchison, Valerie Harpin
}

\begin{abstract}
A postal survey of all computerised children's disability registers in the UK was undertaken in 1996. Information was returned from 155 of 166 districts (93\%). The implementation of computerised special needs registers is a continuing nationwide trend. Although there is evidence of successful use of registers both as an individual and as a population service planning tool, a high percentage of existing registers are functioning in a way which is far short of their potential for research, audit, and planning. Registers which work well have been set up with expertise, have staff enthusiastic about data entry, and are well supported for data output and software modification. There should be continued cooperative work towards a national consensus on the categories of disability and definitions of severity of disability used in these registers. (Arch Dis Child 1998;78:312-315)
\end{abstract}

Keywords: disability; register; special needs

Computerised special needs registers have been developed by health service staff in many districts of the UK over the past 20 years to fulfil four main functions. ${ }^{1-5}$

(1) Service planning; for example determining the numbers of children with severe communication disability who need educational provision in a particular locality.

(2) Epidemiology and research; for example the evaluation of a prenatal screening programme.

(3) Clinical audit; for example using the register to identify a group of children, such as those with incontinence or challenging behaviour, for whom standards of care can be evaluated.

(4) Individual patient care; for example to aid in the coordination of multidisciplinary and interagency case review or planning meetings.

Since 1989 the Children Act has not only made it a duty for social services departments to keep a register of disabled children, but has also asked these departments to work with health service registers where these exist. There is both expectation and scepticism that health services registers can be used for a fifth function:

(5) To enable social services departments to fulfil their Children Act duties.

We are both paediatricians working with a remit to implement special needs registers in our local districts. Informal conversation with paediatric colleagues had left us with the impression that many are dissatisfied with the registers they possessed. Many worked in isolation and expressed the need to share knowledge and good practice. Equally their local social services colleagues had insufficient guidance and worked in relative isolation and inefficiency due to duplication or lack of knowledge.

The aim of the study was to gain an overview of the state of development of NHS children's computerised special needs (disability) registers in the UK. The objectives were: (1) To identify a "lead person" with responsibility for special needs registers in the health service for each district of the UK irrespective of whether a computerised register had actually been implemented in that district. (2) To obtain basic information from each "lead person" about the capability of their system.

\section{Methods}

Postal questionnaires were used in two phases. Questionnaire (A) was sent to community paediatricians in every district to establish:

(1) The name of the person with lead responsibility for a health service special needs register.

(2) If the district possessed or was planning to implement a computerised register.

(3) If they did not, whether they know of another local agency that did.

A more detailed questionnaire (B) was sent to lead paediatricians who reported a register in questionnaire (A). Questionnaire (B) asked specific questions designed to assess register function. Cerebral palsy was chosen as a condition upon which to construct these questions because it is relatively common compared with other diagnoses that can cause disability and has significant resource implications. A system for assessing the severity of motor impairment is in widespread use. ${ }^{6}$

To assess planning capability we asked: "Will your register give names of children with cerebral palsy, by locality, by GP [general practitioner], by school and by severity?" To assess research capability we asked: "Could your register tell you which children with cerebral palsy had a gestation of less than 28 weeks; and which had visual impairment?" To assess capability for service delivery we asked: "For a selected child with cerebral palsy will the register tell you; which services the child receives; the names of therapists; whether the child has an electric wheelchair; and the date of the last multi-disciplinary review?" In these areas we asked how easily the data could be found and how complete they were.

We also asked specifically about support time for the system, its strengths and weak- 
nesses, and if the respondents could give any practical examples of uses or abuses of their systems. We asked about future developments and we requested copies of data collection proformas where available.

\section{Results}

Altogether 166 geographical districts were identified. In some cases these were coterminous with the boundaries of 1974 health districts without corresponding to any present day health commission boundaries.

A total of $155(93 \%)$ returned data on questionnaire (A); 93 of these $(60 \%)$ said they had a computerised register and were therefore sent questionnaire (B). Ninety two of the 93 respondents were paediatricians. Seventy one $(77 \%)$ of the 93 who said they had a computerised register returned questionnaire $\mathrm{B}$ giving more information. The 71 were all doctors. Only three of the 71 forms were incomplete, two because the register had only just been started, and one without information on uses and abuses and strengths and weaknesses.

The earliest computerised register dated from 1975; however $60 \%$ had been started within the previous five years. Many districts without registers reported plans to create a register in the near future (fig 1).

The names of the doctors in each district with a responsibility for registers have been formed into a mailing list with the potential to enable future communication within a "users' group".

Overall there was no information from $7 \%$ of districts. At least $37 \%$ of districts in the UK do not have a computerised children's special needs register. At least $56 \%$ of districts definitely do have such a register.

HARDWARE AND SOFTWARE

Twenty seven (38\%) used a PC with local database. The most commonly specified software systems were Paradox and Access. Forty four $(62 \%)$ use one of the child health systems such as RICHES. Two PC users were about to change to a child health system while two system users were about to change to a PC.

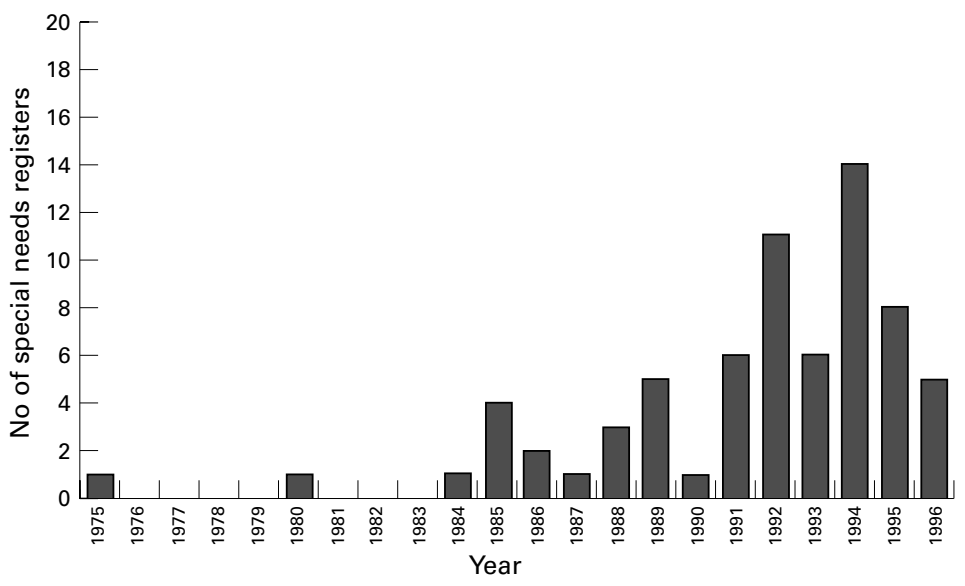

Figure 1 Number of special needs registers started by year.
SYSTEM CAPABILITY: PLANNING

Sixty $(85 \%)$ users could identify children with cerebral palsy by locality; $62(87 \%)$ could identify them by GP. Sixty three $(89 \%)$ could identify them by school but only 41 (58\%) could identify children with cerebral palsy by the severity of their condition.

For locality, school, and severity those reporting "No" were equally likely to use PC as system hardware. The respondents who could not select by GP were all PC users. Overall 25 of 71 said that their data were incomplete. Others reported that the register might not include "milder cases". As far as ease of obtaining the data was concerned, 13 said it would be easy. Seven implied it would be difficult with comments like "can take months" and "report production is slow."

\section{SYSTEM CAPABILITY: RESEARCH}

Thirty nine $(55 \%)$ said their register could identify children with cerebral palsy who had a gestation of less than 28 weeks; $93 \%$ said that the register could tell which children with cerebral palsy had visual impairment. Many of these commented that it would be difficult to do and likely to be incomplete, particularly with respect to milder cases.

\section{SYSTEMS CAPABILITY: SERVICE DELIVERY}

Thirty four ( $44 \%$ ) reported that for a selected child with cerebral palsy the register could tell which services the child received. A further 14 (20\%) said partially; 22 (33\%) said their register could not do this. Fourteen $(20 \%)$ said their register could give the names of the therapists. Many respondents commented that frequent staff changes occurred and therefore no data were collected. Thirty (42\%) reported that for a selected child with cerebral palsy the register could tell whether the child had an electric wheelchair; $40(57 \%)$ said it couldn't. Twenty $(28 \%)$ said that the register could tell the date of the last multidisciplinary review for a selected child with cerebral palsy. $45(67 \%)$ said no. A small number said that the register could give details of medical reviews but not interdisciplinary reviews.

\section{STAFF TIME}

Many respondents were unable to say how many hours per week were dedicated to supporting the register. There were comments such as: "It is part of the clinical work". "It is difficult to guess". "It is not accounted for, it is part of spare time". When administrative times were reported they were from 0 to 2.5 whole time equivalents. Clinical time was reported to vary between 0.5 and 26 hours per week. Two respondents mentioned a nurse as responsible.

\section{STRENGTHS AND WEAKNESSES}

"System" users reported that strengths were: the population base; the integration of the special needs register with other modules of the child health system; the use of similar systems in several districts; the fact that data were both cheap and accessible; and the availability of a mass of data. 
Weaknesses were poor access to the systems and poor program writing support to answer inquiries and produce reports. Many systems were inflexible and not interactive. Some were time consuming and poorly supported by software companies who made heavy charges to make changes to software.

The strengths reported by PC users were that the programs were simple, accessible, and flexible. They could be designed locally for local needs. There was local control and expenses were low. Good links were possible with other local computerised data systems.

Weaknesses reported by PC users were inability to link with other systems and incompleteness of data input. Writing the initial programs could be difficult and more difficulties could arise if the designers of the initial program left the district. Several users thought there were no weaknesses in their PC systems!

USES

We asked for specific examples of use of the system. Replies mentioned function as a planning tool. Examples included respite care services, services for autism, and a motor planning difficulties service. Others discussed using data to influence commissioners, and using registers to arrange reviews of patient care. Eight specifically mentioned use for audit and research. Subjects included autism, cerebral palsy, transition to adulthood and "looked after children" (children for whom a local authority had assumed parental care). They also used the register for answering questionnaires. Thus research questions were sometimes from outside the department or district. A recent example is a national study looking at growth standards for children with Down's syndrome.

ABUSES

Most could give no example of abuses of special needs registers but one reported two examples: a public health medicine department had lost confidential hard copies of a database; doctors had photocopied database printouts without permission and distributed them to other colleagues. One cited underuse of the system as an abuse.

DATA COLLECTION PROFORMAS

There were many similarities between data collection proformas but also great variations. All systems recorded basic demographic data. The degree of additional detail ranged from a total of one side of A4 to extremely detailed "booklets". Where severity coding was used many districts used similar criteria and functional categories. These had often been modelled with local variations on British Association for Community Child Health recommendations. ${ }^{7}$

\section{Discussion}

The aims of computerised special needs registers are: (1) service planning; (2) epidemiology and research; (3) clinical audit; and (4) individual patient care.

A register which cannot identify children with cerebral palsy by locality is hardly likely to be able to serve any useful planning function. Altogether $14 \%$ of existing registers certainly fall into this category and a further $37 \%$ are likely to be dysfunctional due to incomplete data. The assessment of severity is useful for planning in that it will have a crude correlation with service need. Only 58\% had these data and in many cases the database was incomplete. Therefore, considering cerebral palsy alone, over $50 \%$ of existing registers are unable to fulfil planning functions. The figure may be higher if other conditions are chosen or more detailed questions are asked.

In probing the research utility of registers the study looked at the ability to link motor disability to neonatal factors. Only 55\% showed evidence of a link with the simplest of neonatal data. Severity is also useful in research as an outcome measure related to neonatal events and interventions. The present study did not inquire which measures or definitions of severity of motor impairment were used, however many respondents included severity coding definitions with their data entry forms. The degree of concurrence of these coding definitions suggests that it would be possible to converge towards nationally accepted coding guidelines. ${ }^{7}$ If this were achieved there is great potential to inform national work on service delivery and to fuel research into interventions and support for children with disabilities and their families.

Research questions will vary between districts. In choosing a link between cerebral palsy as an example of a diagnosis and core neonatal data we are presuming that particular research data are required. Those districts that cannot make the link might have chosen not to do so. The question for them is whether the system could be set up to deliver this information if required. This survey does not answer that question, though presumably if one register can do it, then any register can be set up to collate this information if given adequate resources. The survey asked about visual impairment and cerebral palsy, estimating that being able to look at multiple impairments and the clustering of impairments might be a common research use. Very few systems are set up to give this information. We cannot be clear whether this shows a basic lack in what should be a core dataset, or whether this reflects the research questions which are actually being asked.

In contrast with research we expected some uniformity of the datasets useful for assessment of service delivery. Altogether $33 \%$ of districts with a register do not collect information about specific service delivery; $80 \%$ do not have the names of individual therapists. Perhaps this is less useful and more likely to need frequent update. In contrast, the use of very expensive health service equipment, such as an electric wheelchair, should surely be known to any register attempting to keep track of service delivery; $58 \%$ do not. Presumably the prescribers know the details but these fragments of information are not collated onto a central register for an overall view of service provision.

Less than $30 \%$ can give the date of the last interdisciplinary review. This probably reflects 
the priorities of those who designed the programs. Certainly here is an opportunity yet to be seized in the majority of registers.

We were gratified to find so little response to requests for examples of system abuse. This should not be an excuse for complacency. The potential for abuse remains. How is one to interpret the absence of allocated administrative time? Some of the reported registers may well be maintained by enthusiasts or as "doctors' toys". System data were described as cheap by some respondents and expensive by others. These seemingly contradictory findings may reflect the availability of in house support whose true cost is hidden. In house support may also make the difference between accessible and inaccessible data in two similar systems.

\section{Conclusions}

Overall our survey shows that the implementation of computerised special needs registers is a continuing nationwide trend but that a high percentage of existing registers are functioning far short of their potential.

There is no indication that better or worse overall performance is due to the choice of PC and commercial database software versus one of the purpose developed child health packages. Success must therefore be due to other factors which we identified but did not quantify in this study and are:

- The expertise that was used to set up the original system.

- The enthusiasm of the staff who collect and enter data.

- The support available to make software modifications.

- The availability of staff with the time and expertise to produce data outputs of use to individual clinicians.

These factors should be clearly considered in the light of local knowledge and skills when setting up new databases.
There should be continued cooperative work towards a national consensus on the categories of disability and definitions of severity of disability used in these registers.

The information collected in this study has potential to support an interactive nationwide users' group. This could be achieved at least partly through the production of a regular users' bulletin or newsletter.

Can we address the sceptical view that registers can't work, don't work, and are an inefficient use of time?

In the minds of many people concerned with the population aspects of disease, a register is a panacea: the establishment of a register will in some mysterious way, resolve hitherto unsolved problems (attributed to Millar).

Such a view is naive in the extreme and has resulted in the failure of registers in the past.

Anyone who has booked an airline ticket knows that where the commercial will exists information technology systems can be made to work well. Over the past five years much energy within the NHS has been spent producing contracting systems for an internal market that has now been abandoned. If only some of those resources could be turned to the further development of clinical information systems there would be a chance to prove the sceptics wrong. Or right.

1 Johnson A, King RA. A regional register of early childhood mpairments: a discussion paper. Community Medicine 1989;11:352-63.

2 Johnson A. Use of registers in child health. Arch Dis Child 1995;72:474-7.

3 Colver AF, Robinson A. Establishing a register of children with special needs. Arch Dis Child 1989;64:1200-3.

4 Woodruffe C, Abra A. A special conditions register. Arch Dis Child 1991;66:927-30.

5 Blair M, Hutchison T. Special needs registers: dreams and realities. In: Spencer N, ed. Progress in community child health. Vol 2. Edinburgh: Churchill Livingstone, 1997: 57-67.

6 Amiel Tilson C, Stewart A. Follow up studies during the first five years of life: a persuasive assessment of first five years of life: a persuasive assessment

7 British Association for Community Child Health. Disability in childhood, towards nationally useful definitions. London: Royal College of Paediatrics and Child Health, 1994. 